\title{
Lack of evidence for diaphragmatic fatigue over the course of the night in obstructive sleep apnoea
}

\author{
J.M. Montserrat, E.N. Kosmas, M.G. Cosio, R.J. Kimoff
}

Lack of evidence for diaphragmatic fatigue over the course of the night in obstructive sleep apnoea. J.M. Montserrat, E.N. Kosmas, M.G. Cosio, R.J. Kimoff. @ERS Journals Ltd 1997.

ABSTRACT: The aim of this study was to determine whether diaphragmatic fatigue develops over the course of the night in patients with obstructive sleep apnoea (OSA).

Patients with severe OSA underwent overnight polysomnography with the addition of gastric and oesophageal catheters for measurement of transdiaphragmatic pressure $(\boldsymbol{P d i})(n=7)$ and a gastro-oesophageal electrode for determination of diaphragmatic electromyogram (EMGdi) $(n=5)$. Analyses of Pdi and EMGdi were performed to detect fatigue during the large inspiratory efforts at the end of apnoeas in Stage 2 sleep at the beginning and end of the night. Measurements included $\boldsymbol{P}_{\mathrm{di}}$ values, shape analysis of the $\boldsymbol{P} \mathrm{di}$ waveform, the relaxation rate $(\tau \mathbf{R})$ of $\boldsymbol{P}_{\mathrm{di}}, \mathbf{E M G d i}$ and its relationship to $P$ di, and the centroid frequency $(f \mathrm{c})$ of EMGdi.

End of apnoeic $P$ di and EMGdi increased from the beginning to end of the night (e.g. 19 $\pm 14 \%$ increase in $P$ di; $p<0.05$ ). The rate of increase in $P$ di and EMGdi during apnoeas did not change. The Pdi versus EMGdi relationship was linear, and remained unchanged over the course of the night. There was no significant change in the shape of the $P_{\mathrm{di}}$ waveform, and there were no changes in $\tau R$ from the beginning to the end of the night $(0.13 \pm 0.01 \mathrm{~s}$ for both periods). There was also no shift in the $f \mathrm{c}$ of the EMGdi power spectrum $(94 \pm 5$ vs $93 \pm 6 \mathrm{~Hz}$; Ns), and no change in the relationship of $f \mathrm{c}$ to $P$ di or EMGdi from the beginning to the end of the night.

These findings do not support the development of diaphragmatic fatigue over the course of the night in obstructive sleep apnoea.

Eur Respir J., 1997; 10: 133-138.
Desmond N. Stoker Sleep Laboratory, Royal Victoria Hospital, McGill University, Montreal, Quebec, Canada.

Correspondence: R.J. Kimoff

Rm. L4.08

Respiratory Division

Royal Victoria Hospital

687 Pine Ave W

Montreal

Quebec

Canada H3A $1 \mathrm{~A} 1$

Keywords: Arousal

fatigue

respiratory muscles

sleep apnoea

Received: December 121995

Accepted after revision October 31996
Patients with obstructive sleep apnoea (OSA) experience repeated episodes of upper airway obstruction during sleep, with resultant hypoxaemia and hypercapnia and progressively increasing inspiratory efforts, until arousal occurs and airway patency is restored. The inspiratory efforts generated at the end of apnoeas are often very large, such that when transdiaphragmatic pressure $(P \mathrm{di})$ and the tension-time index of the diaphragm (TTdi) are determined, end-apnoeic values in some patients approach or surpass the threshold for fatigue described for normal subjects [1-4]. These efforts are generated under conditions of hypoxaemia, hypercapnia and declining cardiac output [5], which are known to predispose to muscle fatigue [6-9]. We, therefore, speculated that inspiratory muscle fatigue could develop during the course of the night in OSA. This could have important pathophysiological consequences for the severity of apnoea, and possibly also for ventilation during wakefulness $[1,2,6,9,10]$.

One previous study found evidence of impaired inspiratory muscle contractility in OSA, in that pleural pressure relaxation rates $(\tau R)$ during voluntary sniff manoeuvres were prolonged in the morning compared with the preceding night prior to sleep [11]. However, the development of inspiratory muscle fatigue during sleep over the course of the night in OSA has not been specifically investigated. The aim of this study was, therefore, to determine whether diaphragmatic fatigue develops from the beginning to the end of the night in a group of severe OSA patients.

\section{Methods}

\section{Subjects}

The data were obtained from seven males with documented OSA, who were recruited from the Royal Victoria Hospital Sleep Disorders Clinic during the course of studies on the mechanisms of lengthening of apnoea during the course of the night [12-14]. Patients were eligible if they had not yet received treatment for OSA, had no other active medical problems, and had no major pulmonary dysfunction on routine pulmonary function testing (forced expiratory volume in one second (FEV1) and forced vital capacity (FVC) $>70 \%$ of predicted). This study was approved by the Human Ethics Committee of the Royal Victoria Hospital, and written informed consent was obtained from all subjects prior to the study. 


\section{Protocol}

The subjects were monitored during one night with standard polysomnographic recording, with the addition of two balloon-tipped catheters (seven subjects) for the measurement of oesophageal $(P$ oes $)$ and gastric $(P$ ga $)$ pressures, and a diaphragmatic electromyographic electrode (EMDdi) (five subjects). Pressure was recorded using differential transducers (Sanborn Model 267BC, Waltham, MA, USA), and $P$ di was obtained as ( $P$ ga $\left.-P_{\text {oes }}\right)$ using a computerized system (CODAS, DATAQ Instruments Inc., Akron, OH, USA). The EMGdi electrode consisted of a modified Swan-Ganz catheter with three silver rings, $2 \mathrm{~mm}$ wide, spaced $18 \mathrm{~mm}$ apart and mounted at the distal end of the catheter. The catheter was anchored at the gastro-oesophageal junction via a balloon at the catheter tip. The EMGdi was obtained from the adjacent electrode pair which yielded the largest amplitude signal. The EMG was conditioned using a Grass amplifier (Model 7P511; Quincy, MA, USA) with band pass filtering between 30 and $1,000 \mathrm{~Hz}$.

Measurements of maximum transdiaphragmatic pressure $(P$ di,max $)$ and maximal EMGdi $(E M G d i, m a x)$ were obtained during wakefulness in the supine position, according to the method of LAPORTA and GRASSINO [15]. During sleep, continuous polysomnographic monitoring (Grass Model 78) was performed using electro-oculographic, electro-encephalographic and submental EMG electrodes for sleep staging, according to standard criteria [16]. Arterial oxygen saturation $\left(\mathrm{Sa}_{\mathrm{a}} \mathrm{O}_{2}\right)$ was measured with a pulse oximeter (Ohmeda Biox 3700, Louisville, CO, USA). Ribcage and abdominal motion were monitored by inductance plethysmography (Respitrace, Ardsley, NY, USA), and airflow was assessed using a thermistor. $P_{\text {oes }}, P$ ga and EMGdi were displayed continuously on the computer screen, and were intermittently transferred to disk and recorded on frequency-modulated (FM) magnetic tape (AR Vetter CO, Rebersburg, PA, USA) for subsequent playback and analysis.

\section{Data analysis}

Data were analysed from two periods of 10 consecutive apnoeas at the start and at the end of the night. The criteria for identifying the periods of analysis were that the patient was in the supine position, during established Stage 2 sleep (with repeated end-apnoeic arousal), with no sustained wakefulness or rapid eye movement (REM) sleep for at least 10 min before or following the apnoeic period. The first period at the start of the night, and latest period at the end of the night which fulfilled these criteria were selected. Continuous sleep (with endapnoeic microarousals) was required between the two analysis periods, in that the aim of the study was to assess the effects of repeated apnoeas during the course of the night.

Obstructive apnoeas were defined as episodes of cessation of airflow of more than $10 \mathrm{~s}$ duration with continued inspiratory efforts. The peak and mean $P$ di for obstructed inspiratory efforts during apnoeas were determined. The inspiratory time $(t \mathrm{I})$ and the total respiratory cycle time ( $t$ tot) were measured from the $P$ di tracing. The diaphragmatic tension-time index (TTdi) for obstructed efforts was calculated in the standard manner as:
$P$ di $/ P$ di,max $\times t \mathrm{I} / t$ tot (4). Other parameters used to assess diaphragmatic fatigue were: 1$)$ the time constant of relaxation $(\tau \mathrm{R})$ of $P \mathrm{di} ; 2)$ the shape characteristics of the $P$ di waveform; 3$)$ the relationship between EMGdi electrical activity and $P$ di; and 4$)$ the centroid frequency $\left(f_{\mathrm{c}}\right)$ of the EMGdi power spectrum. $\tau \mathrm{R}$ and the shape analysis of the $P$ di waveform were determined for the last 2 occluded efforts of apnoeas, and the other parameters were measured for the last three occluded efforts. Values were then compared between the two periods of analysis at the beginning and end of the night.

$\tau \mathrm{R}$ was determined from the $P$ di waveform according to EsAu et al. [17], by analysis using "Anadat" (RHTInfoDat Inc., Montreal, Canada) of a $P$ di tracing acquired at $1 \mathrm{kHz}$ sampling rate. $P$ di curves had to meet the following criteria for analysis: a clear peak pressure; a smooth monotonic decay of pressure from the peak; and a clearly identifiable baseline. Sixty five percent of $P$ di curves met these criteria. There was no difference in the proportion of curves analysed from the beginning compared to the end of the night.

The $P$ di pressure waveform was analysed using the approach of Levine and GiLlen [18]. The $P$ di values were examined as a percentage of peak inspiratory $P$ di at 25 , 50,75 , and $90 \%$ of $t \mathrm{I}$ for each subject. The percentage of $t \mathrm{I}$ at which the peak $P$ di occurred was also determined [18].

For analysis of EMGdi, the raw signal was played back using the CODAS system, with sampling at $1 \mathrm{kHz}$. EMG segments distorted by QRS complexes or detectable motion artefact were deleted [18-20]. The remaining sections of data from a single effort were then divided into 250-350 ms segments, with typically 2-4 segments obtained from each inspiratory effort. Values for each effort were calculated as the mean of the values for all segments of the effort. For each segment, EMG amplitude was determined as the root mean square (RMS) of the raw signal [21] computed using the CODAS system. The power spectrum was computed by applying a Hanning window and Fast Fourier Transform (FFT) at frequencies between 30 and $250 \mathrm{~Hz}$, and the $f \mathrm{c}$ of the power spectrum then determined according to the method of Schweitzer et al. [20], using validated software (Anadat).

For comparison of the relationship between EMGdi and $P$ di during the course of the night, the linear regression relationships [6] between peak $P$ di and EMGdi RMS were determined for the last three obstructed efforts of apnoeas combined, and the slopes and intercepts at the beginning and end of the night were compared. Since $f_{\mathrm{c}}$ may vary with the level of inspiratory effort [22], the relationship between $f_{\mathrm{c}}$ and $P_{\mathrm{di}}$ and between $f_{\mathrm{c}}$ and EMGdi RMS were calculated in the same fashion, and compared across the night. The ratios $f_{\mathrm{c}} / P$ di and $f_{\mathrm{c}} / \mathrm{EMGdi}$ for obstructed efforts in individual subjects were also calculated and these values were compared at the beginning and end of the night.

A paired t-test was used to test for differences between the values for $P$ di and EMG variables at the beginning and end of the night. Linear regression relationships were determined in the standard fashion [23]. Statistical comparison between the two periods of analysis of the slopes and Y-intercepts for linear regression relationships was performed by calculation of the t-statistic, 
using the difference in values divided by the standard error of the difference [23]. A p-value of less than 0.05 was considered statistically significance.

\section{Results}

The subjects in the present study were obese, had normal or only mildly abnormal lung function and were normocapnic. All demonstrated severe OSA, with a group mean apnoea-hypopnoea index of $64.1 \pm 8.8$ (SD) events $\cdot h^{-1}$. Periods of repetitive apnoea during Stage 2 sleep were identified for analysis at the beginning and end of the night, using the criteria described above. The mean time between analysis periods was $3.1 \pm 0.7 \mathrm{~h}$.

\section{Pdi measurements}

In keeping with previous studies [1-3], inspiratory effort, as measured both by $P$ di and TTdi, increased progressively at the end of apnoeas, reaching maximal values just prior to airway opening either on the last $(91 \%$ of apnoeas) or second-to-last (9\% of apnoeas) obstructed effort. There was no difference in this distribution between the two periods of analysis. Apnoea duration increased from the beginning to the end of the night, and the rate of increase in $P$ di during apnoeas did not change. Inspiratory effort at end-apnoea increased significantly from the beginning to the end of the night $(p<0.05)$. The data for the last occluded effort at the beginning and at the end of the night are presented in table 1 , and the $P$ di and TTdi values for the second- and third-last obstructed efforts showed similar changes.

The values for $\tau R$ for the last obstructed effort during apnoeas are shown in table 1 . The values obtained for the second-to-last obstructed effort were identical. There were no significant changes in the relaxation rate of $P \mathrm{di}$ from the beginning to the end of the night.
The results of the shape analysis of the $P$ di waveform are illustrated in fig. 1 and table 2 . There were no significant changes from the beginning to the end of the night in the shape of the $P$ di waveform, as reflected in the mean values for $P$ di at $25,50,75$, and $90 \%$ of inspiratory time $(t \mathrm{I})$, or in the percentage of $t \mathrm{I}$ at which peak $P$ di occurred.

\section{EMGdi measurements}

The EMGdi findings are illustrated in table 1 and figure 2. EMGdi amplitude calculated as RMS and RMS/ RMSmax increased progressively at end-apnoea in concert with $P$ di (fig. 2 a). As for $P$ di, EMGdi for the last obstructed effort was significantly greater at the end than the beginning of the night (table 1). The relationship of $P$ di to EMGdi RMS was linear (r=0.78 (beginning),

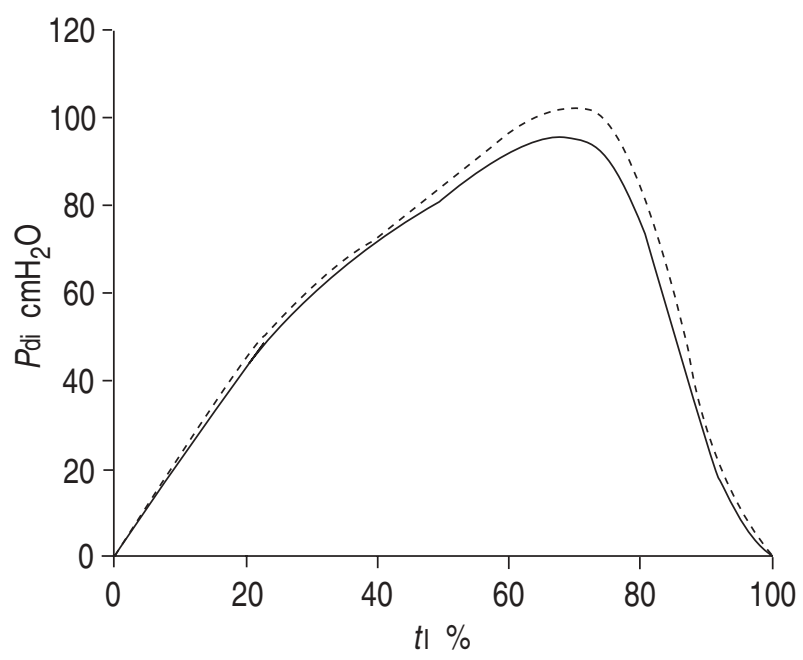

Fig. 1. - Representative tracings from one of the subjects of two transdiaphragmatic pressure $(P \mathrm{di})$ waveforms for the last occluded apnoeic effort at the beginning (- ${ }^{-}$) and at the end (- - - $\left.-{ }_{--}\right)$of the night. $t \mathrm{I}$ : inspiratory time.

Table 1. - Pdi and EMGdi findings during last obstructed effort

\begin{tabular}{|c|c|c|c|c|c|c|}
\hline $\begin{array}{l}\text { Subject } \\
\text { No. }\end{array}$ & Time & $\begin{array}{l}P \text { di peak } \\
\mathrm{cmH}_{2} \mathrm{O}\end{array}$ & TTdi & $\begin{array}{c}\tau \mathrm{R} \\
\mathrm{s}\end{array}$ & EMGdi RMS & $\begin{array}{l}F_{\mathrm{c}} \\
\mathrm{Hz}\end{array}$ \\
\hline \multirow[t]{2}{*}{1} & B & 36.6 & 0.12 & 0.15 & 0.37 & 93 \\
\hline & E & 36.9 & 0.13 & 0.15 & 0.31 & 99 \\
\hline \multirow[t]{2}{*}{2} & B & 29.6 & 0.08 & 0.14 & 0.38 & 85 \\
\hline & E & 32.8 & 0.09 & 0.13 & 0.50 & 85 \\
\hline \multirow[t]{2}{*}{3} & B & 60.9 & 0.18 & 0.11 & 0.65 & 114 \\
\hline & E & 89.0 & 0.23 & 0.10 & 0.75 & 113 \\
\hline \multirow[t]{2}{*}{4} & B & 56.3 & 0.12 & 0.12 & - & - \\
\hline & E & 66.4 & 0.15 & 0.15 & - & - \\
\hline \multirow[t]{2}{*}{5} & B & 41.9 & 0.12 & 0.15 & 0.34 & 93 \\
\hline & E & 49.3 & 0.13 & 0.14 & 0.37 & 76 \\
\hline \multirow[t]{2}{*}{6} & B & 26.6 & 0.12 & 0.12 & 0.51 & 86 \\
\hline & E & 32.3 & 0.16 & 0.13 & 0.57 & 92 \\
\hline \multirow[t]{2}{*}{7} & B & 35.3 & 0.12 & 0.14 & - & - \\
\hline & $\mathrm{E}$ & 42.6 & 0.16 & 0.14 & - & - \\
\hline \multicolumn{2}{|c|}{$\mathrm{B}$ mean $\pm \mathrm{sE}$} & $41.0 \pm 4.9$ & $0.12 \pm 0.01$ & $0.13 \pm 0.01$ & $0.45 \pm 0.10$ & $94 \pm 5$ \\
\hline \multicolumn{2}{|c|}{$\mathrm{E}$ mean $\pm \mathrm{SE}$} & $49.9 \pm 7.9 *$ & $0.15 \pm 0.01 *$ & $0.13 \pm 0.01$ & $0.50 \pm 0.10^{*}$ & $93 \pm 6$ \\
\hline
\end{tabular}

B: beginning of night; E: end of night; $P$ di: transdiaphragmatic pressure; TTdi: diaphragmatic tensiontime index; $\tau$ R: relaxation rate; EMGdi RMS: root mean square of diaphragmatic electromyogram; $f \mathrm{c}$ : centroid frequency of EMGdi. *: p<0.05, B versus $\mathrm{E}$. 
Table 2. - Characteristics of Pdi waveform

\begin{tabular}{|c|c|c|c|c|c|}
\hline Time & $25 \% t \mathrm{I}$ & $50 \% t \mathrm{I}$ & $75 \% t \mathrm{I}$ & $90 \% t \mathrm{I}$ & $\% t \mathrm{I} P$ di,peak \\
\hline \multicolumn{6}{|c|}{ Last occluded effort } \\
\hline B & $50.6 \pm 2.4$ & $72.8 \pm 1.5$ & $77.7 \pm 3.6$ & $28.1 \pm 4.5$ & $58.5 \pm 2.6$ \\
\hline E & $52.1 \pm 2.5$ & $74.8 \pm 0.8$ & $74.2 \pm 3.0$ & $25.5 \pm 3.4$ & $55.2 \pm 2.9$ \\
\hline \multicolumn{6}{|c|}{ Second-to-last occluded effort } \\
\hline B & $52.1 \pm 2.0$ & $80.7 \pm 1.9$ & $91.4 \pm 2.4$ & $26.1 \pm 4.6$ & $70.4 \pm 2.5$ \\
\hline E & $53.8 \pm 2.4$ & $81.7 \pm 1.6$ & $92.1 \pm 2.6$ & $27.1 \pm 5.3$ & $73.4 \pm 2.4$ \\
\hline
\end{tabular}

Values are presented as mean \pm SE for percentage of peak Pdi developed at 25, 50, 75 and 90\% of $t$ I, respectively. B: beginning of night; E: end of night; $\% t \mathrm{I} P$ di,peak: $\%$ of $t \mathrm{I}$ at which peak $P$ di occurred; $t \mathrm{I}$ : inspiratory time; $P$ di: transdiaphragmatic pressure.
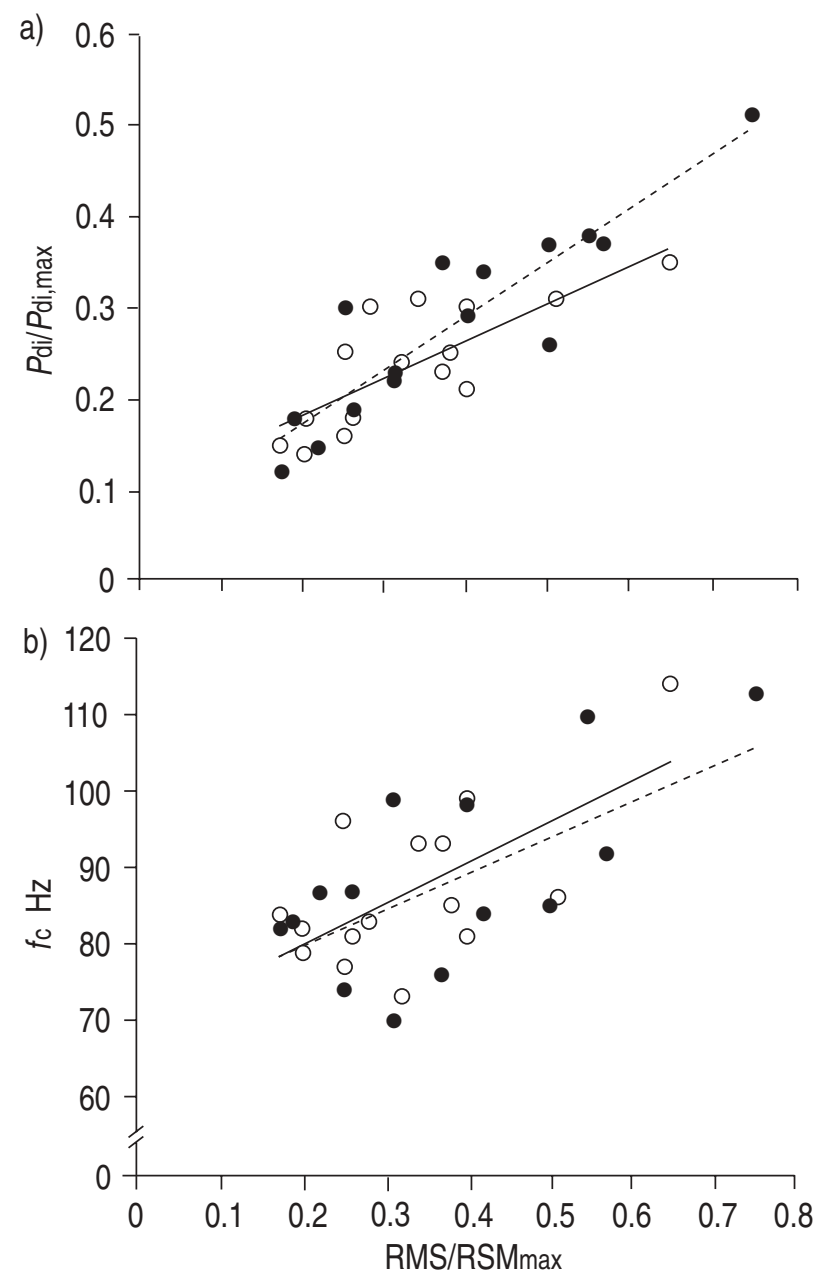

Fig. 2. - Relationship of a) transdiaphragmatic pressure $(P \mathrm{di})$ expressed as $P$ di $/ P_{\mathrm{di}}$ max, and $\left.\mathrm{b}\right)$ centroid frequency $\left(f_{\mathrm{c}}\right)$ to diaphragmatic electromyogram (EMGdi) expressed as RMS/RMSmax. Data points represent the mean values for individual subjects for the last three occluded breaths at the beginning $(\bigcirc)$ and end of the night $(\bullet)$, with solid and dashed lines, respectively, depicting the linear regression relationship for the two periods. Neither the regression relationships of $P$ di $/ P$ di,max vs RMS/RMSmax, nor those of $f_{\mathrm{c}}$ vs RMS/RMSmax differed significantly between the two periods. $P_{\text {di,max: }}$ maxim transdiaphragmatic pressure; RMS: root mean square; RMSmax: maximum root mean square.

$\mathrm{r}=0.90$ (end of the night); $\mathrm{p}<0.001$ for both) (fig. 2a). Neither the slopes nor the intercepts of the relationships were significantly different between the two periods of analysis.

The $f_{\mathrm{c}}$ data are illustrated in table 1 and figure $2 \mathrm{~b}$. The $f$ c showed no statistically significant decrease from the beginning to the end of the night for any of the last three occluded apnoeic efforts. The results for the last occluded effort are shown in table 1 . The $f_{\mathrm{c}}$ values for the third-to-last obstructed effort were $82 \pm 4 \mathrm{~Hz}$ (beginning) and $84 \pm 5 \mathrm{~Hz}$ (end of the night) (NS), and for the second-to-last effort were $85 \pm 4 \mathrm{~Hz}$ (beginning) and $88 \pm 6$ $\mathrm{Hz}$ (end of the night) (Ns). The $f \mathrm{c}$ tended to increase in association with $P$ di, although the linear regression relationship was not statistically significant $(r=0.33$ (beginning), $r=0.48$ (end of the night); $\mathrm{p}>0.05$ for both). However $f_{\mathrm{c}}$ did increase linearly in relation to EMGdi RMS $(\mathrm{r}=0.65$ (beginning), $\mathrm{r}=0.64$ (end of the night); $\mathrm{p}<0.01$ for both) (fig. 2b). There was no significant difference in this relationship between the beginning and end of the night. Comparison of the ratios $f_{\mathrm{c}} / P_{\mathrm{di}}$ and $f_{\mathrm{c}} / \mathrm{RMS}$ for the last three obstructed efforts also showed no significant change over the course of the night.

\section{Discussion}

The aim of this study was to detect whether diaphragmatic fatigue develops over the course of the night in OSA. Muscle fatigue has been defined as the reversible loss of capacity to develop force and/or velocity, resulting from activity under load [9]. Inspiratory muscle fatigue can be produced under experimental conditions when intense respiratory efforts are generated repeatedly against a load $[4,6,9,19]$. Studies in normals have shown that the threshold level of effort for the development of inspiratory muscle fatigue, as measured by TTdi, is 0.15 . We and others have previously shown that TTdi values at end-apnoea are equal to, or surpass, this threshold in some patients [1-3]. In addition, the presence of hypoxaemia, hypercapnia, and reductions in cardiac output have all been shown to predispose to earlier development of fatigue [6-8]. It therefore seemed plausible to propose that inspiratory muscle fatigue could develop over the course of the night in OSA, in which large inspiratory efforts are repeatedly generated against the obstructed airway under asphyxic conditions and in the face of declining cardiac output [5].

The development of respiratory muscle fatigue could be of considerable pathophysiological importance in OSA, both with respect to apnoea severity and ventilatory control during wakefulness. Current evidence suggests that termination of apnoea is associated with arousal, which occurs, at least in part, in response to the generation of a critical level of respiratory effort [2, 13, 14]. If the effort-generating capacity of the muscles declined over the course of the night, there could be delayed time 
to arousal in response to airway occlusion, and thus worsened apnoea-associated blood gas changes. Furthermore, the ventilatory recovery from apnoeas could be impaired by respiratory muscle dysfunction, worsening nocturnal oxygenation with its ensuing complications, and could also contribute to sustained hypoventilation during wakefulness observed in some patients $[1,2,10]$.

The approach to detecting diaphragmatic fatigue in this study was to evaluate $P$ di and EMGdi during the large inspiratory efforts at the end of apnoeas, at the beginning and end of the night. We postulated that if fatigue were produced as a result of repeated apnoeas, the generation of $P$ di during apnoeas and the values for $P$ di at end-apnoea should decline over the course of the night. However, we observed that the rate of increase in $P$ di during apnoeas was unchanged, and the peak $P$ di at end-apnoea, in fact, increased significantly, in association with an increase in apnoea duration over the course of the night. The issue of lengthening of apnoea is discussed in detail elsewhere (12-14). However the finding that generation of inspiratory effort during apnoeas is preserved over the course of the night provides a strong argument against the development of overt inspiratory muscle fatigue. We have observed the same pattern in seven other subjects studied throughout the night with measurement of pleural pressure alone [14]. The pattern, thus, appears to be reproducible, and strongly argues against the loss of inspiratory muscle force-generating capacity during the night.

We also attempted to identify more subtle evidence of diaphragmatic dysfunction using other analyses of $P$ di and EMGdi. EsAu et al. [17] previously reported that changes in the relaxation rate $(\tau \mathrm{R})$ of $P$ di during loaded inspiratory efforts predicts diaphragmatic fatigue. In the latter study, increases in $\tau R$ occurred during fatiguing trials concomitantly with changes in maximal relaxation rate and in EMG high/low (H/L) ratio, prior to the loss of force-generating capacity [17]. Therefore $\tau \mathrm{R}$ of $P \mathrm{di}$ was assessed in an identical manner during end-apnoeic efforts in the present study. No significant changes were found from the beginning to the end of the night, which argues against the development of changes in diaphragmatic contractility over the course of the night.

This finding appears to be at variance with the results of GRIGGS et al. [11], who reported an increase in the $\tau R$ of pleural pressure during voluntary sniff manoeuvres, measured prior to sleep and upon awakening in an apparently similar group of OSA patients. They found no change in maximal mouth pressures from night to morning. The changes in $\tau \mathrm{R}$ were, therefore, interpreted as evidence of impaired inspiratory muscle contractility without development of overt fatigue [11]. Conceivably, their findings may have related to selective changes in contractility of nondiaphragmatic inspiratory muscles identified by assessment of $\tau \mathrm{R}$ using pleural pressure rather than $P$ di [24]. However, this seems unlikely in that in the present study, as previously described [2, 3], $P$ di consisted largely of pleural pressure swings with a relatively minor contribution of $P$ ga. This could be further tested with EMG measurements from nondiaphragmatic muscles [24]. Alternatively, there may be considerable differences in the factors influencing muscle relaxation during a rapid voluntary sniff manoeuvre, compared with during submaximal obstructed efforts produced in response to airway occlusion during sleep under asphyxic conditions. Therefore, we cannot exclude the possibility that similar changes might have been observed in the present patients if sniffs had been performed during wakefulness. However, this was not tested since the focus of this study was on the identification of muscle dysfunction during sleep.

A shape analysis of the $P$ di waveform was performed in the manner described by LEVINE and GILLEN [18]. These authors identified a reduction in the percentage $P$ di swing at 50 and $90 \%$ of $t \mathrm{I}$, without a change in peak $P$ di, in chronic obstructive pulmonary disease (COPD) patients who developed EMGdi signs of fatigue during incremental exercise testing [18]. They therefore proposed that shape changes in the $P$ di waveform are predictive of diaphragmatic fatigue. When we compared $P$ di waveforms using this approach at the beginning and end of the night, no significant change was observed in any of the measures of $P \mathrm{di}$ waveform. This is therefore a further argument against the development of impaired diaphragmatic contractility during the course of the night in OSA.

The relationship between $P$ di and EMGdi activity was assessed in five of the subjects. The development of fatigue is typically associated with a fall in $P$ di developed for a given EMGdi amplitude [6]. In this study, the relationship of $P$ di to EMGdi RMS was linear, both at the beginning and end of the night. In the event of fatigue, the slope of this relationship (fig. 2b) would have been expected to decline during the night [6]. However, there were no statistically significant differences in either the slopes or intercepts of the $P \mathrm{di} v s$ EMGdi RMS relationship between the two periods of analysis.

EMGdi spectral analysis has been widely used to identify early signs of diaphragmatic fatigue [6, 9, 17-20]. One of the most sensitive and reproducible measures for this purpose is the $f_{\mathrm{c}}[19,20]$. In this study, $f_{\mathrm{c}}$ was determined for the last three obstructed efforts during apnoeas at the beginning and end of the night, and no significant differences were found in the mean values for any of the three obstructed efforts between the two periods of analysis. It was also found that $f_{\mathrm{c}}$ varied with the intensity of inspiratory effort, as has been reported previously [22]. The $f_{\mathrm{c}}$ was linearly related to EMGdi RMS and tended also to increase linearly with $P$ di. The $f_{\mathrm{c}}$ vs $P_{\mathrm{di}}$ and $f_{\mathrm{c}}$ vs EMGdi relationships were compared over the course of the night in several ways, in order to detect whether $f_{\mathrm{c}}$ was decreased for a given level of EMG activity or force generation. However, there were no significant changes in either of the relationships over the course of the night.

In view of the relatively small number of subjects studied with EMDdi measurements, there could be concern that the study lacks sufficient statistical power to exclude a false negative result. While one of the subjects showed a decrease in $f \mathrm{c}$ for the last obstructed effort (table 1) from the beginning to the end of the night, the group mean value for this effort did not change significantly. Of note, there was no decrease in $f_{\mathrm{c}}$ for this or any other subject for the third-to-last or secondto-last obstructed effort during the course of the night. Furthermore, the present data indicate that the probability of a Type II error is, in fact, very small. Previous 
studies have identified fatigue via EMG analysis when $f_{\mathrm{c}}$ fell to below 2 sD of baseline values $[18,19]$. In the present study, the average SD for individual subject $f \mathrm{c}$ values for all efforts analysed was $7.5 \mathrm{~Hz}$ at the beginning of the night and $6 \mathrm{~Hz}$ at the end. Thus, to identify fatigue, a decrease in mean $f_{\mathrm{c}}$ of $12-15 \mathrm{~Hz}$ would have been required over the course of the night. Standard power calculations for this magnitude of difference (e.g. 15) between measurements with this degree of variability (e.g. $\mathrm{SD}=7.5)$ for a paired t-test with $\mathrm{n}=5$ yield a power of 0.91 [23]. Thus, we believe the present study has sufficient statistical power to reasonably exclude a true change in $f_{c}$ over the course of the night.

In summary, this analysis of the inspiratory efforts at end-apnoea indicates that there is no loss of force-generating capacity of the diaphragm over the course of the night in severe obstructive sleep apnoea. We also found no evidence from this analysis to support the development of early or "incipient" changes in diaphragmatic contractility, despite the fact that at least four of the subjects attained or surpassed "fatiguing" values of diaphragmatic tension-time index [4] at end-apnoea (table 1). Therefore, it may be that even though potentially fatiguing levels of inspiratory effort are generated repeatedly throughout the night, these are not sustained for sufficiently long periods to result in the development of impaired contractility $[4,6,17,19]$. Thus, as previously proposed by VINCKEN et al. [1], the end-apnoeic arousal response, which leads to resolution of airway occlusion and, thereby, a reduction in inspiratory effort, may provide a defence against the development of inspiratory muscle fatigue in obstructive sleep apnoea patients.

Acknowledgements: The authors would like to thank R. Levy and S.N. Hussain for helpful comments on study design, and C. Sinderby and A. Grassino for their suggestions on EMGdi analysis.

\section{References}

1. Vincken W, Guilleminault C, Silvestri L, Cosio M, Grassino A. Inspiratory muscle activity as a trigger causing the airways to open in obstructive sleep apnea. Am Rev Respir Dis 1987; 135: 372-377.

2. Kimoff RJ, Cheong TH, Olha AE, et al. Mechanisms of apnea termination in obstructive sleep apnea: role of chemoreceptor $v s$ mechanoreceptor stimuli. Am J Respir Crit Care Med 1994; 149: 707-714.

3. Wilcox PG, Paré PD, Road JD, Fleetham JA. Respiratory muscle function during obstructive sleep apnea. Am Rev Respir Dis 1990; 142: 533-539.

4. Bellemare F, Grassino A. Effect of pressure and timing of contractions on human diaphragm fatigue. $J$ Appl Physiol 1982; 53: 1190-1195.

5. E Garpestad, H Katayama, JA Parker, et al. Stroke volume and cardiac output decrease at termination of obstructive apneas. J Appl Physiol 1992; 73: 1743-1748.
6. Roussos C, Bellemare F, Moxham JA. Respiratory muscle fatigue. In: Roussos C, ed. The Thorax. 2nd edn. Part B. New York, Marcel Dekker, 1985; pp. 829-870.

7. Jardim J, Farkas G, Prefaut C, Thomas D, Macklem PT, Roussos C. The failing inspiratory muscles under normoxic and hypoxic conditions. Am Rev Respir Dis 1981; 124: 274-279.

8. Juan G, Calverley P, Talamo C, Schnader J, Roussos C. Effect of carbon dioxide on diaphragmatic function in human beings. $N$ Engl J Med 1984; 310: 874-879.

9. NHLBI Workshop Summary. Respiratory muscle fatigue. Am Rev Respir Dis 1990; 142: 474-480.

10. Sullivan CE, Issa FG. Pathophysiologic mechanisms in obstructive sleep apnea. Sleep 1980; 3: 235-246.

11. Griggs GA, Findley LJ, Suratt PM, Esau SM, Wilhoit $\mathrm{SC}$, Rochester DF. Prolonged relaxation rate of inspiratory muscles in patients with sleep apnea. Am Rev Respir Dis 1989; 140: 706-710.

12. Charbonneau M, Marin JM, Olha A, Kimoff RJ, Levy $\mathrm{RD}$, Cosio M. Changes in obstructive sleep apnea characteristics through the night. Chest 1994; 106: 1695-1701.

13. Montserrat JM, Kosmas EN, Cosio MG, Kimoff RJ. Mechanism of apnea lengthening across the night in obstructive sleep apnea. Am J Respir Crit Care Med 1996; 154: 955-993.

14. Cala SJ, Sliwinski P, Cosio MG, Kimoff RJ. Effect of upper airway anesthesia on apnea duration across the night. J Appl Physiol 1996; 81: 2618-2626.

15. Laporta D, Grassino A. Assessment of transdiaphragmatic pressure in humans. J Appl Physiol 1985; 58: 1469-1476.

16. Rechtschaffen A, Kales A (eds). A manual of standardized terminology, techniques and scoring system for sleep stages of human subjects. Washington, DC, Public Health Service, US Government Printing Office, 1963.

17. Esau SA, Bellemare F, Grassino A, Permutt S, Roussos $\mathrm{C}$, Pardy RL. Changes in relaxation rate with diaphragmatic fatigue in humans. J Appl Physiol: Respirat Environ Exercise Physiol 1983; 54: 1353-1360.

18. Levine S, Gillen M. Diaphragmatic pressure waveform can predict electromyographic signs of diaphragmatic fatigue. J Appl Physiol 1987; 62: 1681-1689.

19. DeVito E, Grassino AE. Respiratory muscle fatigue: rationale for diagnostic tests. In: Roussos $\mathrm{C}$, ed. The Thorax. 2nd edn. Part C. New York, Dekker, 1995; pp. 1857-1879.

20. Schweitzer TW, Fitzgerald JW, Bowde JA, Lynne-Davies P. Spectral analysis of human inspiratory diaphragmatic electromyograms. J Appl Physiol: Respirat Environ Exercise Physiol 1979; 46: 152-165.

21. Basjamain JV, DeLuca CJ. Muscles alive: their functions revealed by electromyography. Fifth edn. Baltimore, Williams \& Wilkins, 1985; pp. 65-100.

22. Hussain SNA, Clement MG, Vanelli G, Albertini M, Agiggini G. The effect of level of contraction on the electromyographic power spectrum of the diaphragm in pigs. Exp Physiol 1991; 76: 765-775.

23. Glantz SA. In: Primer of Biostatistics. Toronto, McGrawHill, 1981.

24. Fitting JW, Bradley TD, Easton PA, Lincoln MJ, Goldman MD, Grassino A. Dissociation between diaphragmatic and rib cage muscle fatigue. J Appl Physiol 1988; 64: 959-965. 